\title{
Gold nanorods: contrast agents for photoacoustic imaging?
}

C. Ungureanu, R. Raja Gopal, T. G. van Leeuwen, S. Manohar D.D.S.

C. Ungureanu, R. Raja Gopal, T. G. van Leeuwen, S. Manohar D.D.S., "Gold nanorods: contrast agents for photoacoustic imaging?," Proc. SPIE 6631, Novel Optical Instrumentation for Biomedical Applications III, 663108 (17 July 2007); doi: 10.1117/12.728091 


\title{
Gold nanorods: contrast agents for photoacoustic imaging?
}

\author{
C. Ungureanu*, R. Raja Gopal, T. G van Leeuwen, S. Manohar \\ Biomedical Technological Institute (BMTI) \& Faculty of Science and Technology (TNW), \\ Biophysical Engineering Group, University of Twente \\ P.O. Box 217, 7500 AE Enschede, The Netherlands;
}

\begin{abstract}
Gold nanorods are seen as possible contrast agents for photoacoustic imaging since they have strong absorption peaks at near-infrared wavelengths. Also they are easy to conjugate with various proteins. If these particles can be conjugated with cancer affinity proteins then these particles can accumulate specifically at a tumor site. By detecting the presence of accumulation of gold nanorods inside the tissue the indirect detection of tumor can be realized. When these particles are irradiated with light pulses of appropriate temporal properties and energy the temperature around these particles can be high enough to induce apoptosis or necrosis in the surrounding cells. In order to use these particles at their full potential we must determine precisely their optical properties. We simulated the optical properties of gold nanorods synthesized by us using the DDSCAT code. The simulated spectra agree qualitatively with the spectra determined using spectrometry and also determined using photoacoustic spectroscopy. Further the values of molar extinction coefficient derived from the simulations were similar to the data measured experimentally by other groups. These results validated qualitatively the model used in the simulations. During simulations we found that the choice of the dielectric function used in simulations plays an important role in the results.
\end{abstract}

Keywords: gold nanorods, contrast agents, photoacoustic imaging, cancer imaging, nanoparticles

\section{INTRODUCTION}

Photoacoustic imaging is a new and promising technique to provide images of living tissue with high resolution. This technique uses a pulsed laser light to induce temperature rise in an absorbing structure inside tissue. The resulting thermoelastic expansion generates an ultrasound wave that is detected by an appropriate ultrasound detector. Information about size, shape, structure and position of the absorbing structure can be determined by analyzing the emitted ultrasound wave. Blood is currently the main photoacoustic source when living tissues is investigated. It has two main advantages:

- Has a high optical absorption coefficient especially in the green region of wavelength.

- Is present in large quantities in tissues.

Cancer is known for its ability to develop high densities of blood vessels through the process of angiogenesis. The presence of these blood vessels provides the contrast in cancer images acquired using optical or photoacoustic imaging. The maximum penetration depth for the light in the green region is limited to a few millimeters due to the high scattering and high absorptions of tissue in this region of wavelengths. To increase the penetration depth, light in the near infrared (NIR) region is required. In this range of wavelengths $(600 \mathrm{~nm}-1100 \mathrm{~nm})$, light can reach depths of few centimeters. However, the absorption coefficient of blood at these wavelengths is small. To compensate this lack in the absorption, contrast agents are required to improve sensitivity of the technique. These contrast agents must be biocompatible, highly specific and strongly optical absorbing substances.

Recently the possibility of using gold nanoparticles as contrast agents in photoacoustic imaging was raised [1, 2]. The optical properties of gold nanoparticles are governed by the collective oscillations of free electrons gas on the their surface (called surface plasmons). These plasmons may oscillate resonantly with light having a specific wavelength. The elongated particles present two plasmon peaks: one transversal due to oscillations of electrons along the short axis and the other one longitudinal due to oscillations along long axis.

* c.ungureanu@tnw.utwente.nl; phone: +31-(0)53-489 3112; fax:+31-(0)53-489 1105 
By changing the size and aspect ratio of these gold nanorods is it possible to tune the longitudinal plasmon peak to any desired wavelength in the red and NIR wavelengths. Using a suitable conjugation of gold nanorods with specific proteins it is possible to accumulate at a cancer site a large number of these particles. The photoacoustic effect generated by the accumulated gold nanorods is likely to be higher than blood, allowing high sensitivity and high specificity images to be made. To be able to use these particles efficiently as contrast agents in photoacoustic imaging, their optical properties must be precisely determined.

We characterized the optical properties of gold nanorods, synthesized in our laboratory [3], using spectrophotometry, photoacoustic imaging and computer simulations.

\section{EXPERIMENTAL SECTION: MATERIAL AND METHODS}

\subsection{Gold nanorod synthesis}

We synthesised gold nanorods using a wet chemical method with silver ion assistance introduced by Nikoobakht and El-Sayed [4].The synthesis process contains two steps:

1. Synthesis of gold seed.

2. Growth of the gold nanorods.

The reagents used for the synthesis of the gold seed and gold nanorods are: tetrachloroauric acid $\mathrm{HAuCl}_{4} .3 \mathrm{H}_{2} \mathrm{O}$ (Acros Organics), CTAB $>99 \%$ (Fluka), sodium citrate (Sigma), sodium borohydride $\mathrm{NaBH}_{4}$ 99\% (Aldrich), ascorbic acid 99\%(Aldrich), silver nitrate $\mathrm{AgNO}_{3}$ 99.8\% (Merck).

\subsubsection{Synthesis of gold seed.}

A solution of CTAB $(5 \mathrm{ml}$ of $0.2 \mathrm{M})$ was sonicated for 20 minutes at $40^{\circ} \mathrm{C}$ in a water bath. A solution of $\mathrm{HAuCl}_{4} \cdot 3 \mathrm{H}_{2} \mathrm{O}(5 \mathrm{ml}$ of $5 \times 10 \mathrm{E}-4 \mathrm{M})$ was added with continuous stirring under an inert atmosphere of nitrogen gas. Then an ice-cold solution of $\mathrm{NaBH}_{4}(0.6 \mathrm{ml}$ of $0.01 \mathrm{M})$ was added at once with vigorous stirring for 1 minute.

\subsubsection{Growing the gold nanorods with various aspect ratios}

The growth solution consists of CTAB $(0.2 \mathrm{M})$ and $\mathrm{HAuCl}_{4} \cdot 3 \mathrm{H}_{2} \mathrm{O}(0.0005 \mathrm{M})$. Two such identical solutions of 5 $\mathrm{ml}$ were prepared. Appropriate volumes of $\mathrm{AgNO}_{3}(0.006 \mathrm{M})$ depending on the desired aspect ratio of nanorods were added to each growth solution. The mild reducing agent ascorbic acid $(0.078 \mathrm{M})$ was added to each solution. Finally, $14 \mu \mathrm{l}$ of 8 minutes aged, preformed CTAB-capped seed solution was added to each conical flask and gently mixed. It was allowed to stand for 3 hours at a temperature of $24^{\circ} \mathrm{C}$. The colour of the nanorod suspensions is dark-blue with a brownish opalescence.

\subsection{Characterization of gold nanoparticles}

Electron microscopy of the nanoparticles was performed using a Zeiss-1550 high-resolution Scanning Electron Microscope (HRSEM). Particle sizes were estimated using NI Vision Module (LABVIEW) on the digital SEM images with at least 250 particles considered in each case. Absorbance spectra of NPs were measured using the Shimadzu PC3101- UV-VIS-NIR spectrophotometer.

\subsection{Optical property simulations}

We used DDSCAT code [5] to simulate the optical properties of the gold nanorods synthesized in our laboratory. This code is based on Discrete Dipole Approximation method first introduced by Purcell and Pennypacker [6]. Briefly, the particle investigated is divided in $N$ polarizable point dipoles, every point dipole is characterized by a polarizability $\alpha_{i}$. When the object is irradiated by a monochromatic wave, each dipole is subjected to a contribution from the incident radiation field and the field re-radiated by all other dipoles. 


$$
\begin{gathered}
E_{i, l o c}=E_{i n c, i}+E_{d i p, i}=E_{0} e^{i k \bullet r_{i}}-\sum_{i \neq j} A_{i j} \bullet P_{j} \\
P_{i}=\alpha_{i} \bullet E_{i, l o c}
\end{gathered}
$$

$P_{i}$ is the dipole moment of the $i$ th element and $A_{i j}$ with $i \neq j$ is an interaction matrix with $3 N \times 3 N$ matrices with elements described as.

$$
A_{i j} \bullet P_{j}=\frac{e^{i k r_{i j}}}{r^{3}{ }_{i j}}\left\{\left\{k^{2} r_{i j} \times\left(r_{i j} \times P_{j}\right)\right\}+\frac{\left(1-i k r_{i j}\right)}{r_{i j}{ }^{2}}\left[r_{i j}{ }^{2} P_{j}-3 r_{i j}\left(r_{i j} \bullet P_{j}\right)\right]\right\}
$$

Using the relations above the extinction cross section and absorption cross section are derived as:

$$
\begin{gathered}
C_{e x t}=\frac{4 \pi k}{\left|E_{0}\right|^{2}} \sum_{i=1}^{N} \operatorname{Im}\left(E_{i, \text { inc }}^{*} \cdot P_{i}\right) \\
C_{a b s}=\frac{4 \pi k}{\left|E_{0}\right|^{2}} \sum_{i=1}^{N}\left\{\operatorname{Im}\left[P_{i} \cdot\left(\alpha_{i}^{-1}\right) * P_{i}^{*}\right]-\frac{2}{3} k^{3}\left|P_{i}\right|^{2}\right\}
\end{gathered}
$$

where $*$ means complex conjugate.

The important input parameters in the simulations are:

- Size

- Shape

- Dielectric function of the nanoparticle

- Local refractive index.

Information regarding the size of the particle is represented by $r_{\text {eff }}$. This is the radius of a sphere with equivalent volume as the gold nanorod. The $r_{\text {eff }}$ for the particles synthesized by us was in the range of $11 \mathrm{~nm}$ to $14 \mathrm{~nm}$. The sizes of the nanoparticles were determined from HR-SEM (High Resolution-Scanning Electron Microscopy) pictures. The shape used in our simulations was the spherically capped cylinder. This geometry is the best representation of the real shape of the gold nanorods as seen from HRSEM pictures. This shape was recently introduced in the geometry libraries from DDSCAT code. For simulations, we used a number of 40000 dipoles [7]. At our effective radius, this number was sufficiently high to achieve convergence in amplitude and wavelength. As dielectric function for gold, we used the data presented in Refs [8] and [9]. The local refractive index used was the refractive index of water equal with 1.33 .

\subsection{Photoacoustic spectroscopy setup}

Photoacoustic theory predicts that the amplitude of the generated ultrasound signal is directly proportional to absorption coefficient of the irradiated substance. The absorption coefficient of gold nanorods is a function of wavelength and concentration. If these particles are irradiated with laser light at different wavelengths and a fixed fluence, then in principle the amplitude of the photoacoustic signal generated by the nanoparticles can follow the same shape as absorption coefficient with wavelength. We irradiate solutions of gold nanorods with a pulsed laser with emission wavelength from $700 \mathrm{~nm}$ to $900 \mathrm{~nm}$ and we measured with an ultrasound transducer the amplitude of the photoacoustic signal generated.

The gold nanorod solution was centrifuged at $10000 \mathrm{rpm}$ for 20 minutes to remove CTAB in the supernatant and the pellet redispersed in demineralized water. The solution was injected in an acoustically and optically transparent 
capillary (Fiber Microdialysis Tube, Spectrum Laboratories Inc) with 0.75 microns inner diameter and 0.95 microns outer diameter.

A Q-switched Nd:YAG laser (Brilliant B, Quantel, France) coupled to an optical parameter oscillator (OPO; OPOTEK, USA) with a pulse duration of $6 \mathrm{~ns}$ at a repetition rate of $10 \mathrm{~Hz}$ was used. Laser light was transmitted to the capillary using an optical fiber with core diameter of $600 \mu \mathrm{m}$ and $\mathrm{NA}=0.22$. The capillary was immersed in water for proper coupling and the photoacoustic signals were detected by a home built double ring sensor [10]. Next, a dual channel digital oscilloscope (TDS220, Tektronix, $1 \mathrm{G} /$ sample/s, 100-MHz bandwidth) collects and transmits the signals to a computer. The oscilloscope was synchronized on the Q-switch trigger generated by the laser. The schematic drawing of the photoacoustic spectroscopy setup is shown in figure 1. The incident energy was approximately $2 \mathrm{~mJ}$.

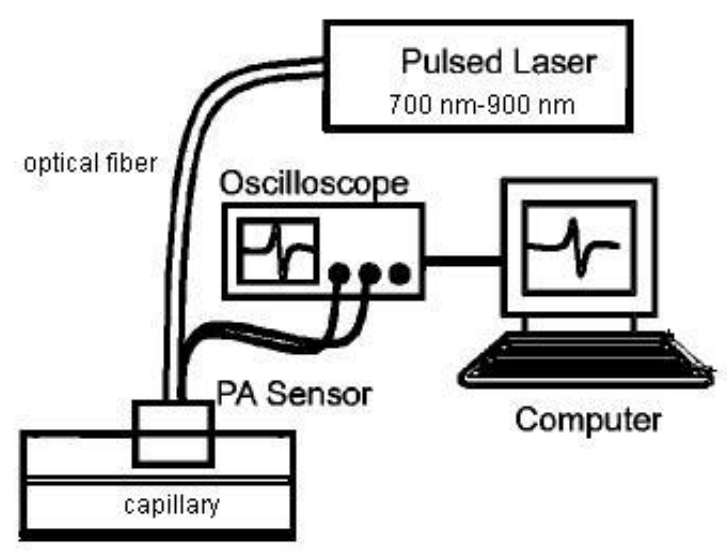

Figure 1. The photoacostic spectroscopy setup.

\section{RESULTS}

\subsection{Synthesis results}

Figure 2.A shows the extinction spectra for two gold nanorods solutions synthesized using the above method and figure 2.B shows a HR-SEM picture of particles synthesis using $100 \mu \mathrm{AgNO}_{3}$, with aspect ratio of 2.85. The form of the nanorods from the image justifies the use of the spherically capped cylinder model in the simulations.

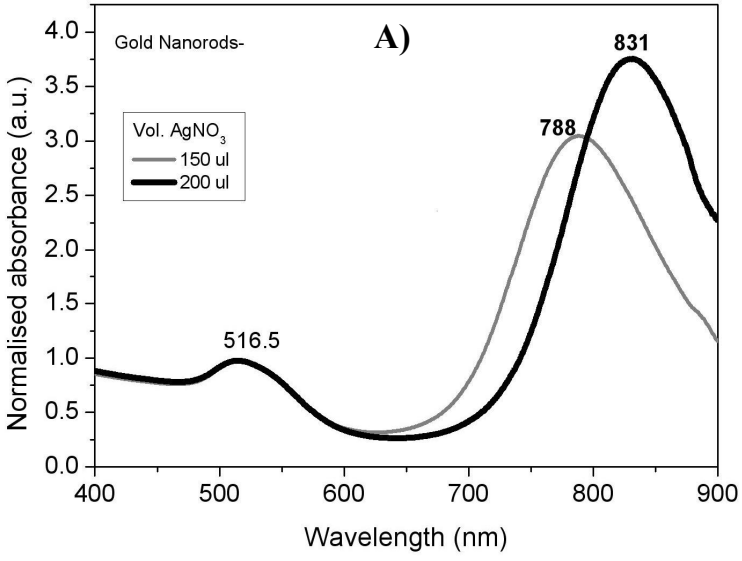

Figure.2 A. Absorbance spectra for particles synthesized using 150 and $200 \mu \mathrm{l} \mathrm{AgNO}_{3}$ normalized at their transverse plasmon peak

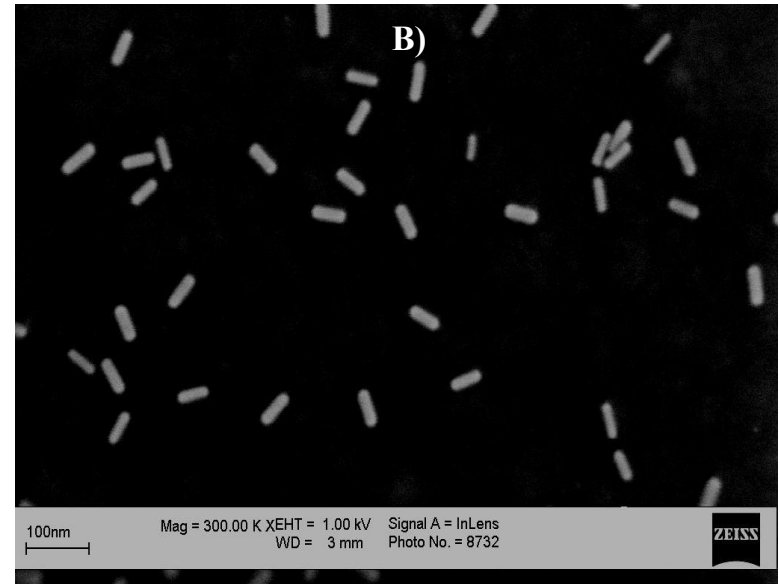

Figure.2.B The HRSEM picture of nanorods synthesized using $100 \mu \mathrm{l} \mathrm{AgNO}_{3}$. 


\subsection{Computer simulations results}

We simulated the optical properties for a gold sphere with $12.5 \mathrm{~nm}$ radius using DDSCAT code and Mie Theory. For these simulations, the dielectric functions of gold from Refs. [8] and [9] were used. Figure 3 shows the simulated extinction spectra using DDSCAT code and Mie theory, and figure 4 shows the normalized experimental and simulated extinction spectrum for a solution of gold spheres with $25 \mathrm{~nm}$ diameter.

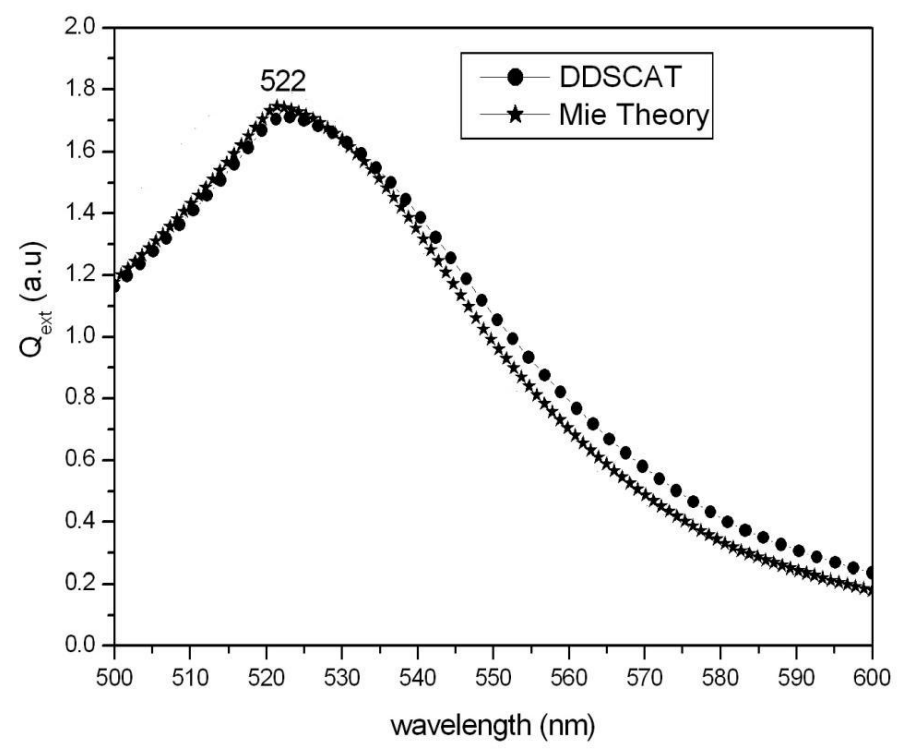

Figure 3. Q Q ext (extinction efficiency) simulated for gold sphere with $25 \mathrm{~nm}$ diameter using Mie theory and DDSCAT code. The dielectric function used for both simulations was data from Ref. 7.

The Mie theory and DDSCAT simulations give similar results in the case of gold spheres.

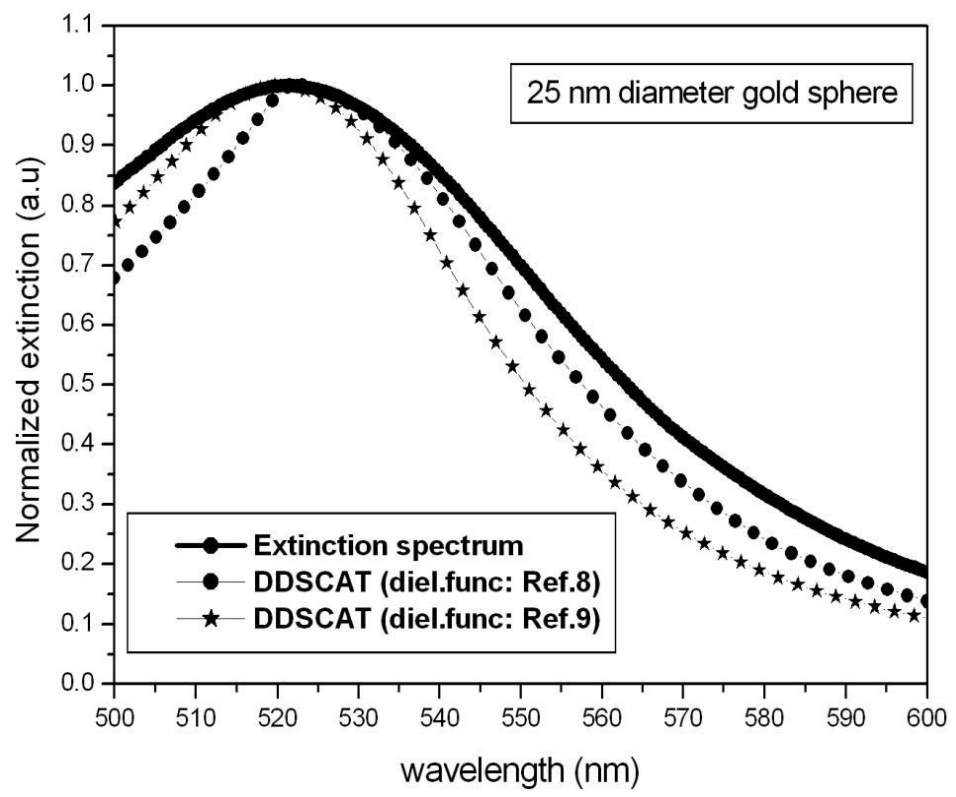

Figure 4. The normalized simulated and experimental extinction spectrum for gold spheres with $25 \mathrm{~nm}$ diameter. 
The computer simulations and the experimental results are in good agreement. The use of the dielectric functions of gold from Ref. 8 and Ref. 9 for DDSCAT simulations of gold nanospheres gives similar results. This is due to the similarity of the dielectric function from the 2 sources in the green region of the spectrum where the plasmon peak of interest occurs.

On the other hand, the dielectric functions from the 2 sources deviates considerably in the NIR region, which is where the logitudinal plasmon peaks of gold nanorods are present. The effect of using the 2 sets of dielectric functions is evident in Fig. 5 with a deviation of up to $30 \mathrm{~nm}$ in the location of the peak. Here only the position of the LP peak of a nanorod with aspect ratio of 3.55 (length $50.4 \mathrm{~nm}$ and width $14.2 \mathrm{~nm}$ ) is shown. The refractive index and number of dipoles used was the same for both simulations.

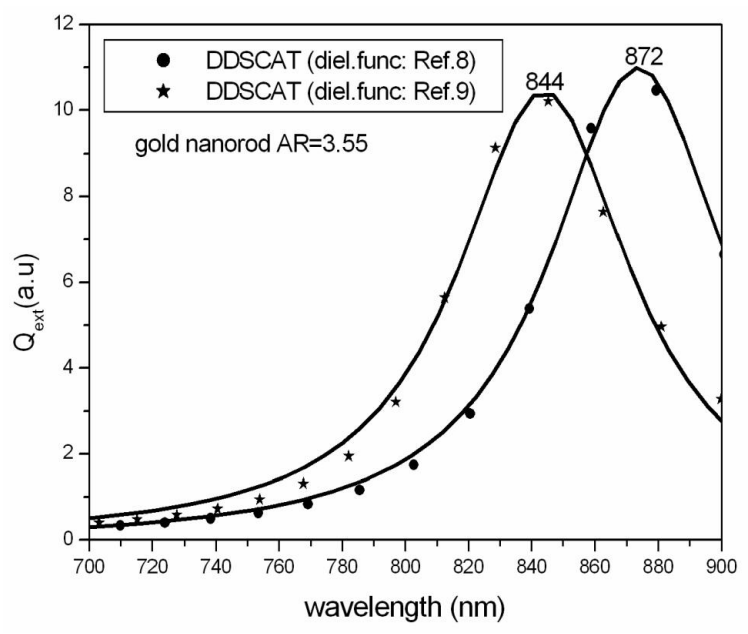

Figure 5. Simulated extinction spectrum for gold nanorod having the same aspect ratio, size, local refractive index but with different dielectric functions. Only the longitudinal plasmon peak is shown.

Using the information presented above we simulate the extinction, absorption and scattering spectra for particles synthesized in our laboratory using the dielectric function presented in Ref. [8]. We used this data because this dielectric function was used to characterize optical properties for particles synthesized using a similar protocol [11]. Figure 6.A shows the experimental and simulated extinction spectrum for particles with aspect ratio of 3.1 normalized in amplitude. The simulated extinction, absorption and scattering spectrum for particles with aspect ratio of 3.1 is shown in figure 6 . B.

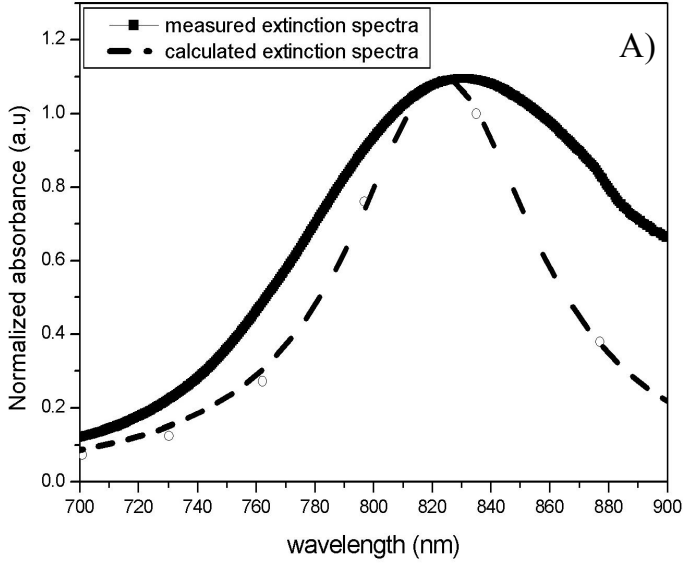

Figure 6.A. Simulated and measured extinction scattering spectrum for particles with aspect ratio of 3.1 aspect ratio. (length of $52 \mathrm{~nm}$ and width of $16.8 \mathrm{~nm}$ ).

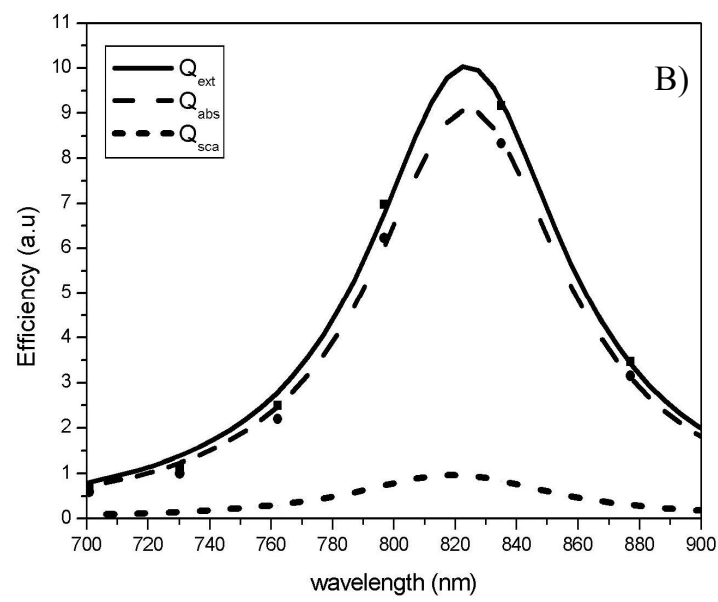

Figure 6.B Simulated extinction, absorption and spectrum for gold nanorods with the 3.1 
In figure 6.B only interval between $700 \mathrm{~nm}-900 \mathrm{~nm}$ is shown for a better visualization. The position of the simulated longitudinal plasmon peak is shifted compared with the one measured experimentally. This difference is mainly due to the uncertainties in dielectric function of gold and the local refractive index. From simulations, we conclude that in the case of our synthesized particles, the absorption is considerably greater than scattering and dominates the extinction of light. Using $C_{e x t}=0.2303 \varepsilon / N_{A}$ where NA is the Avogadro number and $\mathrm{C}_{\mathrm{ext}}$ is the simulated extinction cross section, we calculated a value of $7 \cdot 10^{9} \mathrm{M}^{-1} \mathrm{~cm}^{-1}$ for the molar extinction coefficient for particles with length of $41.7 \mathrm{~nm}$ and width $13.9 \mathrm{~nm}$. This value is slightly higher than the molar extinction coefficients measured experimentally [12] for particles with approximate $r_{\text {eff. }}$.

\subsection{Photoacoustic spectroscopy results}

Figure 7 shows a typical trace of the photoacoustic signal generated by the capillary, which contains gold nanorods. and figure 8 shows the peak-peak amplitude of the photoacoustic signals are plotted together with the extinction spectra measured using the spectrophotometer. The signals were corrected for variation of the energy with wavelength. The photoacoustic spectra agree well with the conventionally determined extinction spectra with the differences in the shape due probably to the non-ideal detector characteristics.

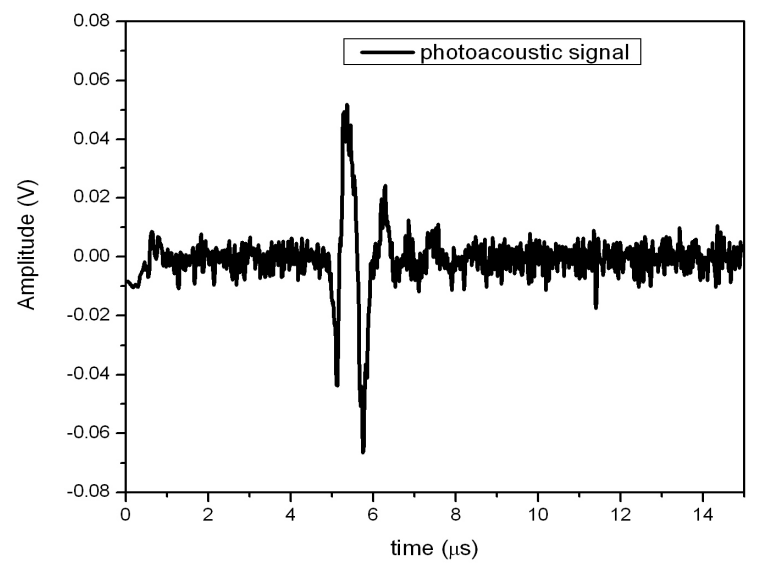

Figure.7. Photoacoustic signal generated by the capillary filled with gold nanorods with the longitudinal plasmon peak at $780 \mathrm{~nm}$.

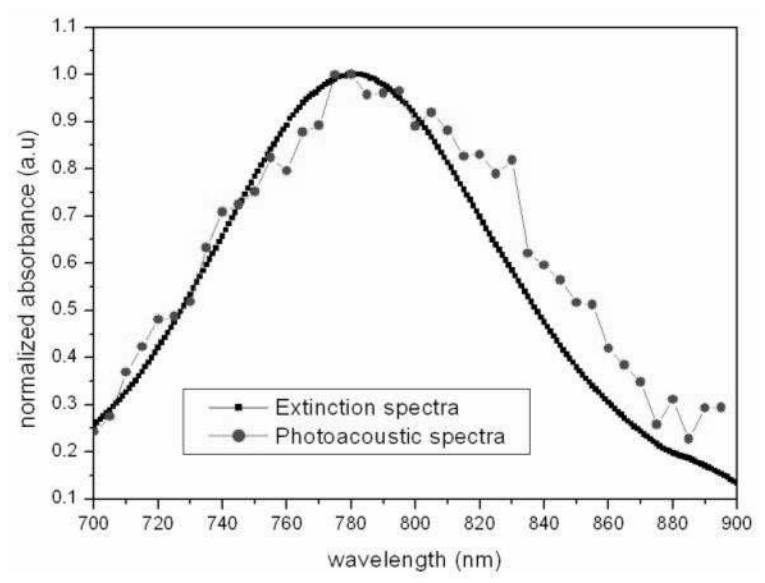

Figure. 8. Normalized extinction and photoacoustic spectra of gold nanorods with the longitudinal plasmon peak at $780 \mathrm{~nm}$. 


\section{DISCUSSION}

The DDSCAT code is useful in simulating the optical properties of gold nanorods. Yet there exist some uncertainties in parameters chosen for the simulations. The refractive index of the embedding medium is usually chosen as that of water while it is known that the rods are covered for the most part by a bilayer of CTAB [13]. Further, it has been speculated that silver may be deposited as a monolayer on the gold surface. This too has not been taken into account in the choice of dielectric function of gold nanorods. These 2 factors may affect the amplitude and the position of the longitudinal plasmon peak of the simulated optical properties of the gold nanorods synthesized using the surfactant assisted $\mathrm{AgNO}_{3}$ based synthesis protocols. Further, there are differences in the dielectric function of gold in the literature $[8,9]$, which are pronounced at NIR wavelengths and affect the location of the longitudinal plasmon peak. We are currently investigating these issues [7].

A qualitative check in testing the suitability of choice of dielectric function for the simulations is comparing the optical interaction efficiency spectra with either experimental spectrophotometric and photoacoustic extinction spectra. Using dielectric functions from Ref. 8 we obtain a close coincidence of the simulated LP peak and experimentally derived peak. Further, the molar extinction coefficient calculated using this value of dielectric function matches compares favorably with values measured experimentally for similar gold nanorods by other researchers [12].

From the simulations we arrive at the optical extinction efficiency which when combined with the geometrical cross-section allows the estimation of the extinction cross-section $\left(C_{e x t}\right)$ in $\mathrm{m}^{2}$. From the experimentally derived extinction coefficient $\left(\mu_{e x t}\right)$ using the spectrophotometer, the number density $(\rho)$ of particles can be estimated as:

$$
\rho=\mu_{\text {ext }} / C_{\text {ext }}
$$

For the nanorods synthesized, we obtain regularly number densities of the order of $10^{10}-10^{11}$ particles per ml.

Further, we can make a prediction of whether contrast enhancement for photoacoustic imaging would be possible. Using the knowledge that the absorption is the major contributor to extinction, we can approximate $C_{a b s}$ $\approx C_{e x t}$. The number of particles required to achieve contrast enhancement can now be calculated as following: if a tumor has an absorption coefficient of $\mu_{a b s}=0.008 \mathrm{~mm}^{-1}$ at $800 \mathrm{~nm}$ then the number of particles required at a tumor site to be greater than the tumor absorption coefficient is $N_{p} \geq \mu_{a} / C_{a b s}$ where the $C_{a b s}$ is $5 \times 10^{-15} \mathrm{~m}^{2}$ at $675 \mathrm{~nm}$ for particles with $44.8 \mathrm{~nm}$ length and $19.8 \mathrm{~nm}$ width. The required number can be smaller than $1.6 \times 10^{9} \mathrm{NR} / \mathrm{cm}^{3}$ if during the photoacoustic imaging process the thermal non-linearity enhancement mechanism (1) is obtained.

The fact that the extinction is dominated by the absorption means that a major portion of light is absorbed and assuming that the efficiency of non-radiative deexcitation is high implies that a large percentage of the incident energy is converted in to heat. This suggests that these particles can be used in photothermal induced therapeutic applications like: local hyperthermia induction, cell lysis due to other effects such as of shock waves and bubble cavitation [14].

\section{CONCLUSION}

We synthesized gold nanorods with various sizes and aspect ratios and we simulated their optical properties using DDSCAT code. The position of the longitudinal plasmon peak from simulations and experimental measurements is found to be shifted slightly. This shift is due to the uncertainties regarding the dielectric function of the gold as well as due to the local refractive index. However, the calculated value for molar extinction coefficient is similar with the value measured experimentally for similar particles. From simulations, we conclude that the extinction of light by the nanorods synthesized by us is dominated by the absorption. This recommends these particles for applications where high optical absorption is required.

The shape of the photoacoustic spectrum matches the extinction spectrum and indicating that at this size of the nanorods, the absorption peak coincide with the extinction peak. These results confirm qualitatively the validity 
of the theoretical model used to simulate optical properties. Using this technique the shape of the absorption spectrum for different particles can be determined. This technique can be helpful in the case of bigger particles like gold nanoshells where the absorption, scattering and extinction spectrum are different.

\section{ACKNOWLEDGMENTS}

The authors thank Prof. Bruce Draine for his invaluable help in introducing the spherically capped cylinder geometry in the DDSCAT code. Dr. Roy Kolkman is acknowledged for the use of the photoacoustic setup. This research is supported by the University of Twente in the thrust area program NIMTIK, and by the Netherlands Organisation of Scientific Research (NWO) and the Technology Foundation (STW) through project TTF.6527. The work was partially sponsored by the National Computing Facilities Foundation (NCF) for the use of supercomputer facilities, with financial support from the Netherlands Organization for Scientific Research (NWO).

\section{REFERENCES}

1. A. A. Karabutov, E. V. Savateeva and A. A. Oraevsky, "Optoacoustic Supercontrast for Early Cancer Detection," Proccedings of SPIE, Vol 4256, pp. 179-187, (2001).

2. M. A. Eghtedari, J. A. Copland, V. L. Popov, M. Motamedi, N. Kotov, and A.A. Oraevsky, "Bioconjugated gold nanoparticles as a contrast agent for optoacoustic detection of small tumors," Proceedings of SPIE, Vol. 4960, pp.76-85, (2003).

3. R. G. Rayavarapu, W. Petersen, C.Ungureanu, J. N. Post, T.G. van Leeuwen, S Manohar," Synthesis and bioconjugation of gold nanoparticles as potential molecular probes for light-based imaging techniques", $I$. $J$. Biomed. Imaging, in press.

4. B. Nikoobakht and M. A. El-Sayed, "Preparation and Growth Mechanism of Gold Nanorods (NRs) Using Seed-Mediated Growth Method," Chem. Mater, vol. 15, no. 10, pp. 1957-1962, (2003).

5. B. T. Draine and P. J. Flatau, 2004, "User Guide to the Discrete Dipole Approximation Code DDSCAT 6.1," http://arxiv.org/abs/astro-ph/0409262v2.

6. E. M. Purcell and C.R. Pennypacker, "Scattering and absorption of light by non-spherical dielectric grains," $J$. Astrophysical., vol. 186, pp. 705-714, (1973).

7. C. Ungureanu, R. G. Rayavarapu, S. Manohar and T. G van Leeuwen, "Influence of choice of dielectric function on DDSCAT simulations: particular case gold nanorods", Manuscript in preparation.

8. P.B. Johnson and R.W. Christy, "Optical Constants of the Noble Metals," Phys. Rev. B, vol. 6, Issue 12, pp. 4370-4379, (1972).

9. D. W. Lynch, W. R. Hunter. In Handbook of Optical Constants of Solids; Palik, E. D., Ed.; Academic Press: New York, pp 350-356, (1985).

10. R. G. M. Kolkman, E. Hondebrink, W. Steenbergen, T. G van Leeuwen and F. F. M de Mul, "Photoacoustic imaging with a double-ring sensor featuring a narrow aperture" J. Biomed. Optics 9 (6), 1327-1335, (2004).

11. K. J. Prashant, L. S. Kyeong, I. H. El-Sayed, and M. A. El-Sayed, "Calculated Absorption and Scattering Properties of Gold Nanoparticles of Different Size, Shape, and Composition: Applications in Biological Imaging and Biomedicine," Phys. Chem. B, 110 (14), pp.7238 -7248, (2006).

12. J. C. Orendroff and C. J. Murphy, "Quantitation of Metal Content in the Silver-Assisted Growth of Gold Nanorods," J. Phys. Chem. B., vol. 110, no. 9, pp. 3990-3994, (2006).

13. B. Nikoobakht and M. A. El-Sayed, "Preparation and Growth Mechanism of Gold Nanorods (NRs) Using Seed-Mediated Growth Method," Chem. Mater, vol. 15, no. 10, pp. 1957-1962, (2003).

14. P. Z. Vladimir and G. Valentin, "Photothermal detection of local thermal effects during selective nanophotothermolysis," Appl. Phys. Lett. 83, 4897 (2003). 